\title{
DYNAMICS OF FOUR-PLANETARY GEARING BOX
}

\author{
L. Pust ${ }^{*}$, L. Pesek ${ }^{* *}$, A. Skuderova ${ }^{* * *}$
}

\begin{abstract}
Planetary gearboxes are more complicated than parallel-axis gear transmission systems and therefore they need deeper dynamic analysis, which is important for reduction of noise and vibration. The main advantage of planetary gearings is the splitting of force flow into several planet wings, but it can be devaluated by unequal load sharing on planet stages. For improving, the floating sun wheal and flexible pins of planets are applied. Dynamic model of such a gearing box is derived in the paper and it is shown that the frequency spectrum contains many multiple eigen-frequencies. Examples of several eigen-modes and transfer functions of this four-planetary gearing box are presented as well. The problem of dimensional nonhomogeneous vectors and matrix is also mentioned. .
\end{abstract}

Keywords: planetary gearing, frequency spectrum, multiple eigenvalues, eigen-modes, transfer functions

\section{Introduction}

In this paper, the solution of spectral properties of the plane type of gearings with four planetary subsystems and with fixed planet carrier is presented. As the all wheels have helical gearings, fluctuation of teeth contact stiffness over a mesh cycle can be neglected. Steady contacts in gearings are asserted by means of preloading due to the constant moment loads on the sun and on outer rig gears.

The experimental investigation of the four planetary gearing box is prepared in our institute and this analytical and numerical research is intended as the basic information for evaluation of the experimental gained data. Therefore is the presented paper oriented on four problems: a) Calculation of frequency spectrum of 18 DOF system, b) ascertaining modal spectrum in digital form and graphical presentation of individual eigen-modes, c) calculation and graphical presentation of transfer functions among individual elements of planet gearings, d) physical interpretation of gained numerical data.

\section{Investigated system}

The structure of the four planetary gearing box is shown in Fig. 1. The computing model simplified on the plane motion of all wheels has 18 DOF consisting from 12 translations $y, x$ [m] and 6 rotations [rad].

Coordinate vector $\mathbf{q}$ describing position of all wheels is

$$
\mathbf{q}=\left[y_{1} x_{1} \varphi_{1}{ }^{1} y_{2}{ }^{1} x_{2}{ }^{1} \varphi_{2}{ }^{2} y_{2}{ }^{2} x_{2}{ }^{2} \varphi_{2}{ }^{3} y_{2}{ }^{3} x_{2}{ }^{3} \varphi_{2}{ }^{4} y_{2}{ }^{4} x_{2}{ }^{4} \varphi_{2} y_{3} x_{3} \varphi_{3}\right]^{T} \text {. }
$$

The positions and directions of individual coordinates are shown in Fig. 1. The pitch radii of the gearing wheels are $r_{1}=0.0585 \mathrm{~m}, r_{2}=0.117 \mathrm{~m} \mathrm{a}_{3}=0.2925 \mathrm{~m}$. The gearing wheels have masses $\mathrm{m}_{1}=25 \mathrm{~kg}, \mathrm{~m}_{2}=$ $50 \mathrm{~kg} \mathrm{a} \mathrm{m}_{3}=256 \mathrm{~kg}$.

Moments of inertia of these wheels are

$\Theta_{1}=0.04 \mathrm{kgm}^{2}, \Theta_{2}=0.45 \mathrm{kgm}^{2}, \Theta_{1}=18.2 \mathrm{kgm}^{2}$.

\footnotetext{
* Ing. Ladislav Pust, DrSc. Institute of Thermomechanics, Dolejskova 5; 182 00, Prague; CZ, pust@it.cas.cz

** Ing. Ludek Pesek, CSc. Institute of Thermomechanics, Dolejskova 5; 182 00, Prague; CZ, pesek@it.cas.cz

*** Ing. Alena Skuderova, Ph.D., Institute of Thermomechanics, Dolejskova 5; 182 00, Prague; CZ, skuder@it.cas.cz
} 
Support stiffness of wheel axes are $k_{1}=1.0 \mathrm{e}+5 \mathrm{~N} / \mathrm{m}, k_{2}=1.2 \mathrm{e}+8 \mathrm{~N} / \mathrm{m}$ and $k_{3}=1.08 \mathrm{e}+8 \mathrm{~N} / \mathrm{m}$.
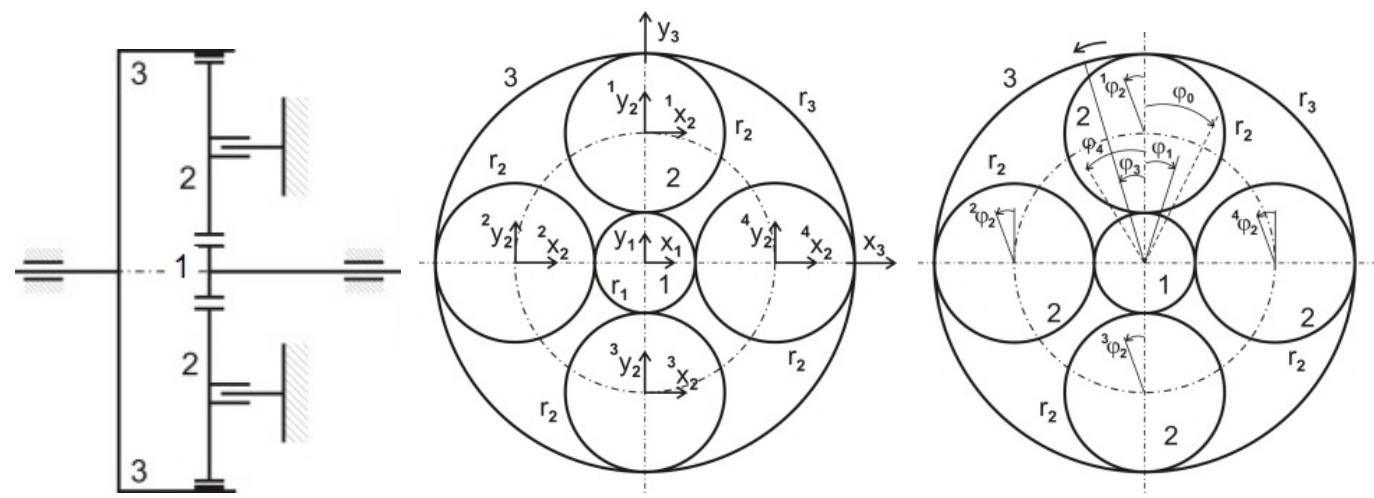

Fig. 1: Structural schema of four-planetary gearings box.

The mean mesh stiffness in the gearing contacts are $k_{12}=3.8 \mathrm{e}+9 \mathrm{~N} / \mathrm{m}$ and $k_{23}=3.9 \mathrm{e}+9 \mathrm{~N} / \mathrm{m}$.

\section{Equations of motion - free vibrations}

The equations of free undamped vibrations can be written in the matrix form

$$
\mathbf{M q}+\mathbf{K q}=\mathbf{0},
$$

where the coordinate vector $\mathbf{q}$ is defined by eq. (1). The inertia matrix $\mathbf{M}$ is square diagonal

$$
\mathbf{M}=\left[\backslash m_{1}, m_{1}, \Theta_{1}, m_{2}, m_{2}, \Theta_{2}, m_{2}, m_{2}, \Theta_{2}, m_{2}, m_{2}, \Theta_{2}, m_{2}, m_{2}, \Theta_{2}, m_{3}, m_{3}, \Theta_{3} \backslash\right]
$$

and it is again dimensional nonhomogeneous with masses $m[\mathrm{~kg}]$ and moments of inertia $\Theta\left[\mathrm{kgm}^{2}\right]$. Stiffness matrix $\mathbf{K}$ is square full, type $18 \times 18$ and again dimensional nonhomogeneous, as it contains elements with dimensions $[\mathrm{m} / \mathrm{N}]\left(\right.$ e.g. $\left.k_{1}, k_{2}\right)$ or with dimensions $\left[\mathrm{m}^{2} / \mathrm{N}\right]$ or $\left[\mathrm{m}^{3} / \mathrm{N}\right]\left(\right.$ e.g. $r_{2} k_{2}$, or $\left.r_{1} r_{2} k_{2}\right)$. Introducing these both matrices into eq. (2) and using special program "eigWik18DOF_240118" elaborated in the system Matlab we gain frequency spectrum [Hz] of investigated four-planet gearing box shown in Tab. 1.

Tab. 1: Eigen-frequency spectrum.

\begin{tabular}{ccccccccc}
\hline $\mathrm{f}_{1}$ & $\mathrm{f}_{2}$ & $\mathrm{f}_{3}$ & $\mathrm{f}_{4}$ & $\mathrm{f}_{5}$ & $\mathrm{f}_{6}$ & $\mathrm{f}_{7}$ & $\mathrm{f}_{8}$ & $\mathrm{f}_{9}$ \\
\hline 0 & $\mathbf{1 7 5 . 3}$ & $\mathbf{1 7 5 . 3}$ & 197.4 & $\mathbf{5 4 2 . 3}$ & $\mathbf{5 4 2 . 3}$ & $\mathbf{8 7 0 . 4}$ & $\mathbf{8 7 0 . 4}$ & $\mathbf{1 0 1 4 . 5}$ \\
\hline $\mathrm{f}_{10}$ & $\mathrm{f}_{11}$ & $\mathrm{f}_{12}$ & $\mathrm{f}_{13}$ & $\mathrm{f}_{14}$ & $\mathrm{f}_{15}$ & $\mathrm{f}_{16}$ & $\mathrm{f}_{17}$ & $\mathrm{f}_{18}$ \\
\hline $\mathbf{1 0 1 4 . 5}$ & 1806.5 & $\mathbf{2 1 6 7 . 2}$ & $\mathbf{2 1 6 7 . 2}$ & 2208.0 & 2368.3 & $\mathbf{3 3 6 5 . 9}$ & $\mathbf{3 3 6 5 . 9}$ & 5574.4 \\
\hline
\end{tabular}

The first eigen-frequency has zero value and corresponds to the revolution of all gearing wheels. The remaining seventeen non-zero eigen-frequencies correspond to the vibrations superposed on this rotation. There are six twofold frequencies and five single frequencies.

The used program „eigWik18 DOF_240118“ in Matlab system ascertains corresponding modes of vibrations in digitalized form. In order to have the possibility of easy analysis of dynamic properties of investigate system, the graphical presentation of eigen-modes is needed. Therefore the specialized computing program "emodeDF240118" in Matlab was elaborated. By means of this program are illustrated, as examples, four lowest eigen-modes of four-planetary gearing box in Fig. 2. 


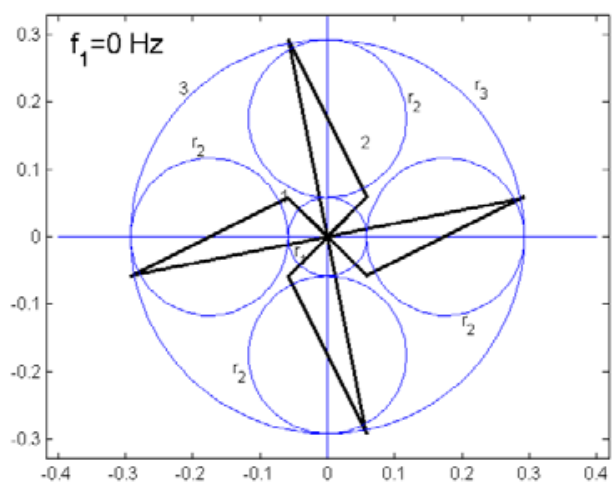

a)

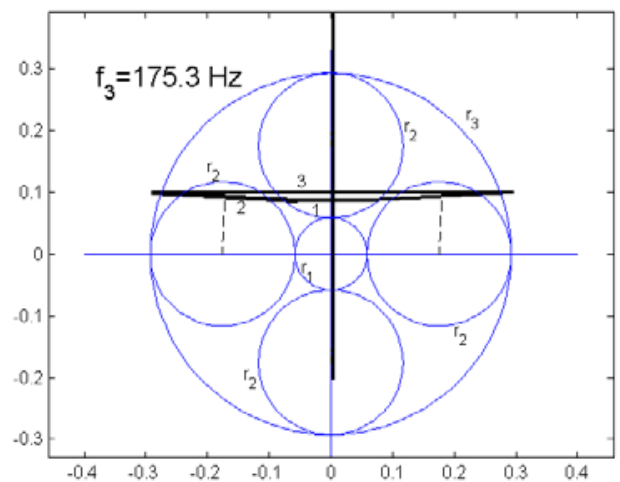

c)

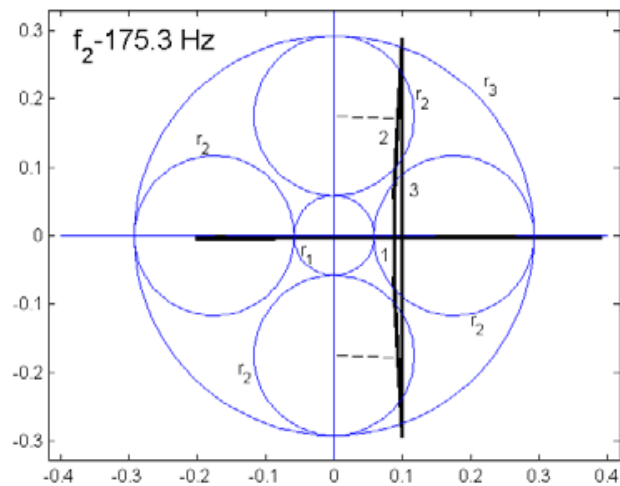

b)

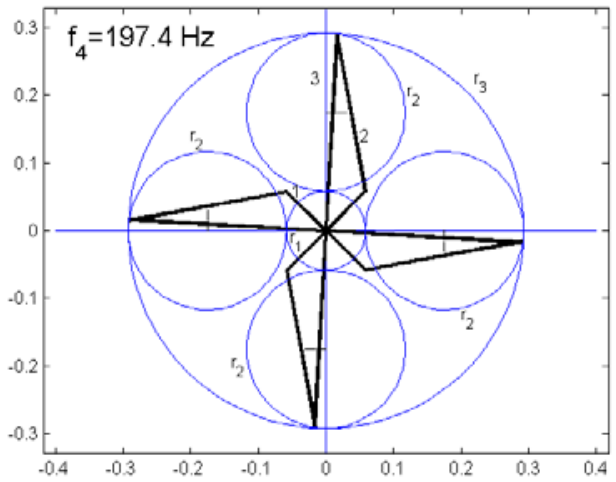

d)

Fig. 2: Modes of free vibration

The Fig. 2a at zero frequency corresponds to the revolution of all gearing wheels. The inclinations if lines are proportional to the angular velocity of individual wheels. Fig. 2b) and 2c) are modes belonging to the twofold eigen-frequency $175.3 \mathrm{~Hz}$. These modes are mutually orthogonal. Fig. 2d is mode of singular eigen-frequency $197.4 \mathrm{~Hz}$ and describes vibrations superposed on the wheel's rotation.

\section{Equations of motion - forced vibrations}

The equations of forced vibrations of investigated four-planet gearing box can be written in the matrix form

$$
\mathbf{M} \ddot{\mathbf{q}}+\mathbf{B} \dot{\mathbf{q}}+\mathbf{K q}=\mathbf{F} \cos (\omega t),
$$

where the damping matrix $\mathbf{B}$ contains the coefficients of linear damping, column vector $\mathbf{F}$ contains amplitudes of excitation forces. The forced vibrations of such a complicated 18 DOF system depend on very many input parameters. Let us use the matrix $\mathbf{H}$ of transfer functions among system elements as the general characteristics of this system. This transfer function matrix has $18 \times 18=324$ elements, some of which have zero value.

Due to the dimensional non-homogeneity of vectors and matrices in the equation of forced vibrations (3) the non-zero elements in the transfer functions matrix $\mathbf{H}$ are also non-homogeneous and can be divided into four group: A) elements with dimension $[\mathrm{m} / \mathrm{N}], \mathrm{B}$ ) elements with dimension [rad/N], C) elements with dimension [m/Nm], D) elements with dimension [rad/Nm].

In the following Fig. 3 there are shown examples of transfer functions. The first number in individual curve description ascertains place of excitation according to order of element in coordinate vector $\mathbf{q}$ (e.g. $\left.1-. .=\mathrm{y}_{1}, 2-. .=\mathrm{x}_{1}, ..\right)$, the second number gives the place of measurement again according order in $\mathbf{q}$. Fig. 3a describes responses in six coordinates on the unit force vertical excitation of central wheel. Fig. 3b presents responses in eight points on the unit horizontal excitation of the first planet. In Fig 3c) are angular responses on force excitations of central (1-.., 2-..) and of ring (16-.., 17..) wheel. The subfigure 


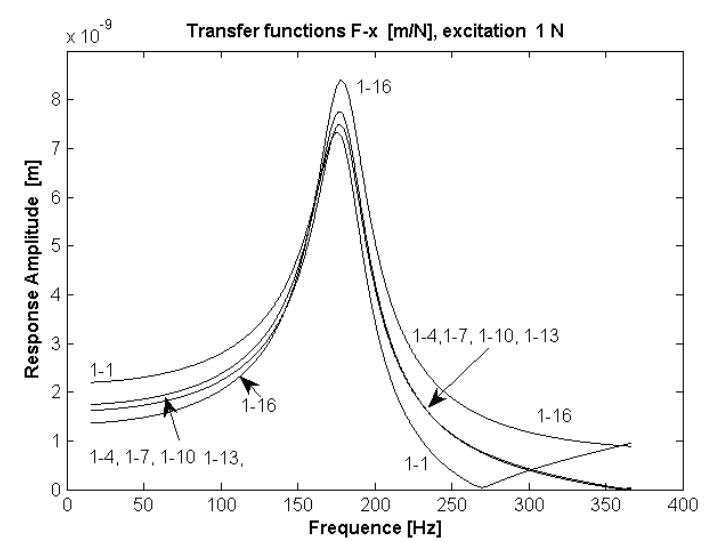

a)

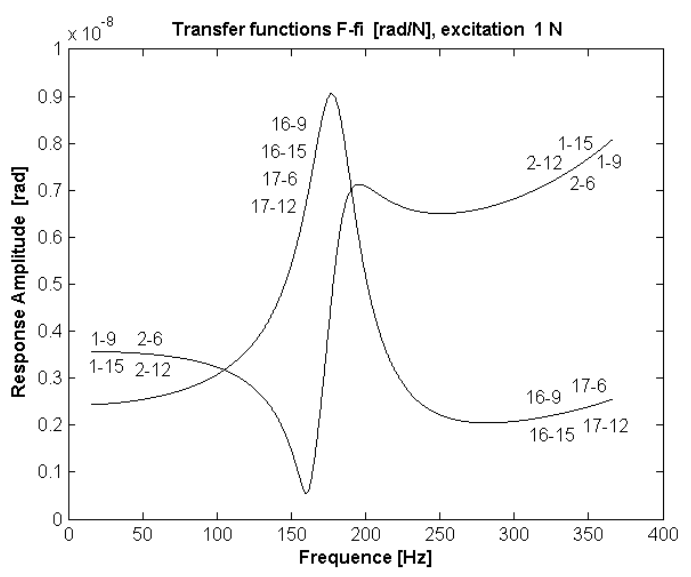

c)

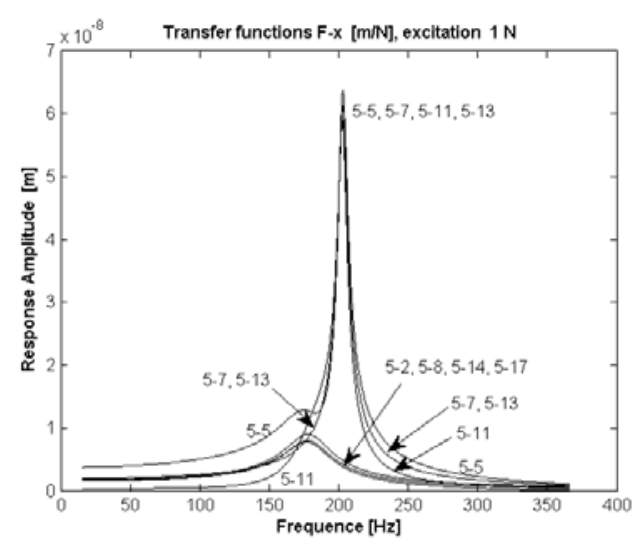

b)

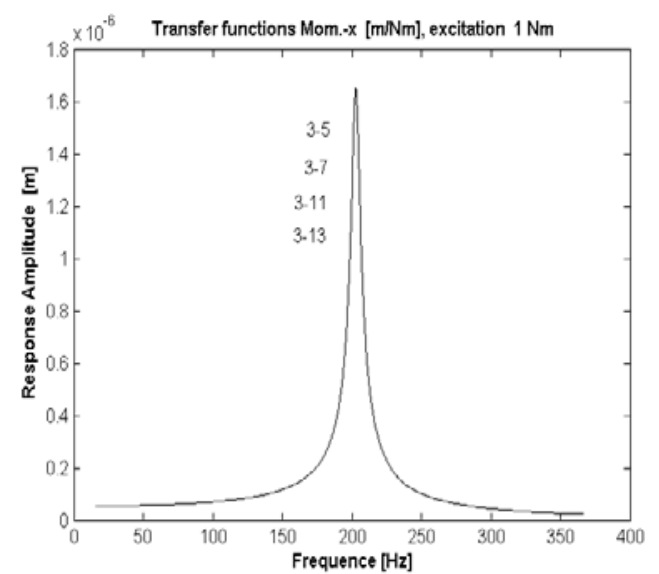

d)

Fig. 3: Examples of transfer functions

Fig. 3d) contains displacement responses on angular moment excitation of sun wheel. From all figures it is evident that one curve can present several responses.

\section{Conclusion}

Frequency and modal spectra together with transfer functions of a gearing box are analysed on computational model with 18 DOF. The investigated gearing box is four planetary type with the fixed planets' carrier. All gearing wheels are supposed to be solid elastically supported bodies with general plain motions. The mesh contacts in gearings are computed as elastic and constant with neglecting the time fluctuation of teeth contact stiffness as in the helical and sufficiently wide gearings is this fluctuation very small. The physical interpretation of numerically gained data is shown on examples of selected elements of transfer functions matrix.

\section{Acknowledgement}

The work has been supported by the project of the IT ASCR AVOZ2076919 "Experimental and theoretical research of planetary gearing with fixed planet carrier”.

\section{References}

Pust, L. and Pesek, L. (2017) Dynamics of planetary gearing box, in: Proc. Dynamical Systems, Theory and Applications - DSTA 2017 T3 (eds. Awrejcewicz, J. and Olejnik, P.), ARSA, Lodz, pp. 473-482.

Pust, L. and Pesek, L. (2016) Free vibration modal spectrum of planetary gearing box, in: Proc. Computational Mechanics (ed. Zeman V.), ZCU, Plzen, pp. 105-106. 